\title{
Comparison of recombinant MPB70 and SahH and a native 20-kDa protein for the detection of bovine tuberculosis by ELISA
}

\section{Yun Sang Cho ( $\nabla$ choys@korea.kr)}

Animal and Plant Quarantine Agency https://orcid.org/0000-0003-1346-7067

\section{Sang Eun Lee}

Animal and Plant Quarantine Agency

Jong-Tae Woo

Gyeonggi Province Veterinary Service Center

Jinsik Oh

Bionote Co., LTD.

\section{Hwan Won Choi}

ChoongAng Vaccine Laboratory

Jin Hyeok Kwon

Animal and Plant Quarantine Agency

Jeong-Tae Kim

Gyeonggi Province Veterinary Service Center

\section{Gunwoo $\mathrm{Ha}$}

Bionote Co., LTD.

\section{Chanhee Park}

Bionote Co., LTD.

\section{Sukchan Jung}

Animal and Plant Quarantine Agency

\section{Jong Man Kim}

Animal and Plant Quarantine Agency

\section{Research article}

Keywords: Mycobacterium bovis, enzyme-linked immunosorbent assay, MPB70, SahH, purified protein derivative

Posted Date: February 21st, 2020

DOI: https://doi.org/10.21203/rs.2.24167/v1

License: (c) (i) This work is licensed under a Creative Commons Attribution 4.0 International License. Read Full License 


\section{Abstract}

Background Bovine tuberculosis (bTB) is a zoonosis mainly caused by Mycobacterium bovis. Test-and-cull protocols and gross pathological examinations of abattoir animals as well as milk pasteurisation have been implemented for preventing the spread of tuberculosis from animals to humans worldwide, including in the Republic of Korea. Despite the importance of precise and rapid diagnostic tests, conventional methods, including intradermal skin tests and $\gamma^{-}$ interferon assays, are limited by the high rate of false negative results for cattle in the late infectious stage as well as laborious and time-consuming procedures. Therefore, antibody detection methods, such as enzyme-linked immunosorbent assay (ELISA), are urgently needed to supplement established approaches and to expand the diagnostic window. In this study, we developed a bTB ELISA by evaluating candidate recombinant and native proteins and various assay parameters.

Results We produced recombinant MPB70 and SahH (rM70S) and a native 20-kDa protein (20K). The 20K ELISA showed $94.4 \%$ sensitivity and $98.2 \%$ specificity and had an optimal sample-to-positive (S/P) ratio cut-off of 0.531 . rM70S ELISA showed $94.4 \%$ sensitivity and $97.3 \%$ specificity with an S/N ratio cutoff of 1.696 .

Conclusion The assays showed the same sensitivity but the specificity was higher for 20K ELISA than for rM70S ELISA. Both assays had acceptable diagnostic efficiency and are expected to be useful for bTB diagnosis in combination with established methods for herd screening and for expanding the diagnostic window.

\section{Background}

Bovine tuberculosis (bTB) is caused by Mycobacterium bovis, which can infect both humans and other taxa, including cattle $[1,2]$. In South Korea, the frequencies of bTB in cattle were $0.08 \%$ (2,898 heads) at the individual level and $0.41 \%$ (427 farms) at the farm level in 2018

(https://www.kahis.go.kr/home/lkntscrinfo/selectLkntsOccrrnc.do,http://library.mafra.go.kr/skyblueimage/28195.pdf). Infected cattle are subjected to test-and-cull and compensation. Accordingly, the economic losses due to bTB in South Korea are high, in addition to the labour-intensive process of bTB diagnosis by national control agencies [3-5].

Furthermore, the potential spread from M. bovis-infected cattle to owners and workers is an important issue[6-10], even though a human M. bovis infection has not been reported to date in South Korea.

Currently, test-and-destroy and abattoir surveillance as well as milk pasteurisation are used for preventing the transmission of animal tuberculosis from animals to humans in South Korea $[4,5,11]$. bTB has been detected by cellmediated immunity-based diagnosis (CEMID), including intradermal skin tests (IST) and $y$-interferon assays, based on the cell-mediated immune reaction to $M$. bovis $[2,12,13]$. However, CEMID has a bTB diagnostic window with a high false-negative rate and is laborious, requiring two farm visits for injection and interpretation, retesting at bTB-positive farms, and evaluations of non-reactors by IST [14-16]. To complement the CEMID, methods for the humoral immunitybased diagnosis (HUMID) of bTB are urgently needed.

In this study, we produced recombinant MPB70 and SahH (rM70S) and compared it with a native 20-kDa protein and a purified protein derivative (PPD) to establish a serological bTB assay with high sensitivity and specificity. To the best of our knowledge, this is the first evaluation of the combination of MPB70 and SahH antigens for bTB ELISA.

\section{Results}

\section{Protein profile and antigenicity}


The major protein component of PPD was a 20-kDa protein, but the overall protein pattern was complex (Fig. 1). The 20-kDa protein of PPD is a major immunoreactive protein against M. bovis-positive serum. Compared with culture filtrate proteins (CFP), PPD had no proteins of larger than $20 \mathrm{kDa}$. However, the immunoreactive protein of CFP was only $20 \mathrm{kDa}$, similar to PPD. Other protein fractions from M. bovis were also analysed by SDS-PAGE and western blotting, including insoluble proteins (INS) and soluble proteins (SOL). The patterns of INS proteins were all diffuse, and the major SOL proteins were 20 and $40 \mathrm{kDa}$. The pattern of INS proteins in PPD was very different from that of CFP, while the pattern of SOL proteins was similar to that of CFP. Of INS. A 40-kDa protein showed the highest immunoreactivity by western blotting with M. bovis-positive serum. However, immunoreactive proteins were not detected in SOL. We also analysed M. avium Johnin, M. avium PPD, and M. phlei PPD. No protein bands were detected for M. avium Johnin PPD, and the major protein bands of M. phlei PPD were $22 \mathrm{kDa}$ and $40 \mathrm{kDa}$. The three sources from M. avium and M. phlei did not show immunoreactive proteins against M. bovis-positive serum.

We targeted a 20-kDa major immunoreactive B cell antigen, MPB70. The 20-kDa antigen was purified at two laboratories, the Animal and Plant Quarantine Agency (APQA) and ChoongAng Vaccine Laboratory (CVL), by anion exchange chromatography [5]. We evaluated the size and protein profile by SDS-PAGE and antigenicity by western blotting (Fig. 1B). Non-reactive proteins with molecular weights exceeding 20 kDa were removed by purification.

rM70S was produced and evaluated with respect to size and antigenicity against M. bovis-positive serum (Fig. 1C). The sizes of MPB70 and SahH were $37 \mathrm{kDa}$ and $75 \mathrm{kDa}$, respectively. The immunoreaction of the recombinant rMPB70 was higher than those of other recombinant proteins, such as ESAT6, HspX, PhoS, MPB64, Antigen 65, and SahH. The 37-kDa MPB64 and MPB70, 30-kDa Antigen 65, 75-kDa SahH, and 20-kDa PPD were clearly immunoreactive against M. bovis-positive serum.

\section{ELISA for the purified protein derivative}

We evaluated 18 bTB-positive serum samples and 975 bTB-negative serum samples. The bTB-positive serum samples were confirmed for $\mathrm{M}$. bovis infections by microbiological isolation, microscopic diagnosis, histopathological findings, and genetic diagnosis. We analysed the results of ELISA using the PPD antigen based on the sample/positive (S/P) ratio, sample/negative (S/N) ratio, and optical density (OD). The average OD value for $\mathrm{M}$. bovis-positive serum samples $(n=18)$ was 0.860 , while that of M. bovis-negative serum samples $(n=975)$ was 0.273 (Fig. 2$)$. Compared with other ELISA antigens, such as M70S and 20K, the reactivity of PPD ELISA differed little between positive and negative sera. The differentiation efficiency of PPD based on the OD value was the lowest among the three ELISA antigens. However, PPD ELISA exhibited an equivalent efficiency to those of 20K and M70S ELISA based on OD values and receiver operating characteristic (ROC) curves (Fig. 3). The average $\mathrm{S} / \mathrm{N}$ ratio of $\mathrm{M}$. bovis-positive serum samples $(n=18)$ was 2.026 , while that of M. bovis-negative serum samples $(n=975)$ was 0.886 . Similar to the comparison of OD values, the difference in the S/N ratio between positive and negative serum samples was the narrowest for PPD among the three ELISA antigens. The differentiation efficiency of PPD by the S/N ratio was the lowest among the three ELISA antigens. The ROC curve for PPD ELISA based on the S/N ratio showed the lowest diagnostic efficiency (Fig. 3). The average S/P ratio for $M$. bovis-positive serum samples $(n=18)$ was 0.958 , while that of $M$. bovis-negative serum samples $(n=975)$ was -0.146 . Unlike the comparisons of OD values and $S / N$ ratios, the difference in the S/P ratio between positive and negative serum samples for PPD was intermediate among the three ELISA antigens. The differentiation efficiency of PPD based on the S/P ratio was intermediate. The ROC curve for PPD ELISA based on the $\mathrm{S} / \mathrm{P}$ ratio showed the lowest diagnostic efficiency (Fig. 3). Optimal cutoffs for the OD value, S/N ratio, and S/P ratio were $0.618,1.289$, and 0.271 , respectively (Table 2 ). The OD value was the most appropriate criteria for PPD ELISA in terms of sensitivity (77.8\%), specificity (98.8\%), positive predictive value (PPV) $(53.8 \%)$, and negative predictive value (NPV) (99.6\%). 
Table 1

Primer sequences of MPB70 and SahH

\begin{tabular}{|c|c|c|c|}
\hline Target gene & Primer (F/R) & Oligonucleotides primer sequence & PCR product size (bp) \\
\hline \multirow[t]{2}{*}{ MPB70 } & Forward (BamHI) & 5'GCGGATCCGGCATGAAGGTA3'a & \multirow[t]{2}{*}{600} \\
\hline & Reverse (Sall) & 5'CCGTCGACTTAAACGCCGGAGGCATTAGCAC3'b & \\
\hline \multirow[t]{2}{*}{$\mathrm{SahH}$} & Forward (BamHI) & 5'GGATCCATGACCGGAAATTTGGTC3'a & \multirow[t]{2}{*}{1,488} \\
\hline & Reverse (Sall) & 5'GTCGACTTAGTAGCGGTAGTGGTC3'b & \\
\hline \multicolumn{4}{|c|}{ a BamHI recognition sequence was underlined. } \\
\hline
\end{tabular}

Table 2

Sensitivity, specificity, positive predictive value, negative predictive value at optimal cutoff according to bTB ELISA antigens

\begin{tabular}{|lllllllll|}
\hline Antigen & Criteria & Cutoff & Sensitivity & Specificity & PPV & NPV & Accuracy & AUC $(95 \%$ Cl) \\
\hline PPD & OD & 0.618 & 77.8 & 98.8 & 53.8 & 99.6 & 98.4 & $0.989(0.981-0.998)$ \\
\hline & S/N & 1.289 & 94.4 & 85.7 & 10.9 & 99.9 & 85.9 & $0.946(0.914-0.977)$ \\
\hline S/P & 0.271 & 94.4 & 94.1 & 22.7 & 99.9 & 94.1 & $0.979(0.958-1.000)$ \\
\hline OD & 1.149 & 83.3 & 97.8 & 41.7 & 99.7 & 97.6 & $0.991(0.983-0.998)$ \\
\hline & S/N & 2.139 & 94.4 & 97.4 & 40.5 & 99.9 & 97.4 & $0.991(0.984-0.998)$ \\
\hline S/P & 0.531 & 94.4 & 98.2 & 48.6 & 99.9 & 98.1 & $0.994(0.988-1.000)$ \\
\hline & OD & 1.320 & 77.8 & 99.5 & 93.3 & 97.9 & 97.5 & $0.990(0.979-1.001)$ \\
\hline & S/N & 1.696 & 94.4 & 97.3 & 77.3 & 99.4 & 97.0 & $0.994(0.986-0.998)$ \\
\hline
\end{tabular}

\section{0-kDa ELISA}

The average OD value for M. bovis-positive serum samples $(n=18)$ was 2.376 , while that of M. bovis-negative serum samples $(n=975)$ was 0.310 (Fig. 2). Compared with other ELISA antigens, such as PPD and rM70S, the reactivity of 20K ELISA exhibited the greatest difference between positive and negative sera. The differentiation efficiency of $20 \mathrm{~K}$ based on the OD value was the highest among three ELISA antigens. The ROC curve for 20K ELISA based on OD values showed the highest diagnostic efficiency (Fig. 3). The average $\mathrm{S} / \mathrm{N}$ ratio for M. bovis-positive serum samples ( $\mathrm{n}$ $=18$ ) was 5.138, while that of M. bovis-negative serum samples $(n=975)$ was 0.671. Similar to the comparison of OD values, the reactivity of $20 \mathrm{~K}$ ELISA based on the $\mathrm{S} / \mathrm{N}$ ratio exhibited the greatest difference between positive and negative sera among the three ELISA antigens. The differentiation efficiency of $20 \mathrm{~K}$ by S/N ratio was the highest among three ELISA antigens. The ROC curve of 20K ELISA based on S/N values showed the highest diagnostic efficiency (Fig. 3). The average S/P ratio for M. bovis-positive serum samples $(n=18)$ was 1.869 , while that of $M$. bovis-negative serum samples $(n=975)$ was -0.174 . Similar to the comparisons of OD values and $S / N$ ratio, the 
reactivity of 20K ELISA based on the difference in the S/P ratio between positive and negative sera was higher among the three antigens. The differentiation efficiency of $20 \mathrm{~K}$ by the S/P ratio was the highest among the antigens. The ROC curve for 20K ELISA based on S/P values exhibited the highest diagnostic efficiency (Fig. 3). Optimal cutoffs for the OD value, S/N ratio, and S/P ratio were $1.149,2.139$, and 0.531 , respectively (Table 2 ). The S/P ratio was the most appropriate criterion for 20K ELISA in terms of sensitivity (94.4\%), specificity (98.2\%), PPV (48.6\%), and NPV (99.9\%).

\section{MPB70 and SahH ELISA}

Average OD value for M. bovis-positive serum $(n=18)$ was 2.224 , while that of M. bovis-negative serum $(n=975)$ was 0.533 (Fig. 2). Compared with the other ELISA antigens, such as PPD and 20K, the difference in reactivity of rM70S ELISA between positive and negative sera was similar but slightly less than that of 20K ELISA and greater than that of PPD ELISA. In M. bovis-positive cattle, the difference in OD values between rM70S and 20K was not significant ( $p=$ 0.623). The differentiation efficiency of M70S based on OD values was similar to that 20K ELISA in bTB-positive cattle. The ROC curve for rM70S ELISA based on OD values showed similar diagnostic efficiency to that of 20K ELISA (Fig. 3). The average $S / N$ ratio of $M$. bovis-positive serum samples $(n=18)$ was 3.788 , while that of M. bovis-negative serum samples $(n=975)$ was 0.748 . Similar to the comparison of OD values, the reactivity of rM70S ELISA based on the difference in $\mathrm{S} / \mathrm{N}$ ratios between positive and negative serum samples was intermediate among the three ELISA antigens. In M. bovis-negative cattle, the difference in the $\mathrm{S} / \mathrm{N}$ ratio between $\mathrm{rM70S}$ and $20 \mathrm{~K}$ was not significant $(p=$ 0.230 ). The differentiation efficiency of $20 \mathrm{~K}$ based on the $\mathrm{S} / \mathrm{N}$ ratio was the highest among the three ELISA antigens. The ROC curve for M70S ELISA based on the S/N ratio showed a similar diagnostic efficiency to that of 20K ELISA (Fig. 3). The average S/P ratio for M. bovis-positive serum samples $(n=18)$ was 0.561 , while that of M. bovis-negative serum samples $(n=975)$ was -0.068 . Similar to the comparisons of OD values and $S / N$ ratios, the difference in reactivity of rM70S ELISA based on the S/P ratio between positive and negative serum samples was the narrowest among the three ELISA antigens. The differentiation efficiency of rM70S based on the S/P ratio was the lowest among antigens. The ROC curve for rM70S ELISA based on the S/P ratio showed the highest diagnostic efficiency, similar to that of 20K ELISA (Fig. 3). Optimal cutoffs for the OD value, S/N ratio, and S/P ratio were 1.320, 1.696, and 0.143, respectively (Table 2). The S/N ratio was the most appropriate criterion in M70S ELISA in terms of sensitivity (94.4\%), specificity (97.3\%), PPV (77.3\%), and NPV (99.4\%).

\section{Discussion}

We compared three bTB ELISA antigens confirmed as highly reactive to M. bovis-specific antibodies. In particular, we used PPD as a crude protein antigen mixture, $20 \mathrm{~K}$ as a purified protein antigen, and $\mathrm{rM70S}$ as a recombinant protein antigen. rM70S and 20K were more sensitive and specific in bTB ELISA than PPD. Interestingly, rM70S, which showed high sensitivity and specificity, was composed of a new combination of antigens for bTB serological diagnosis, MPB70 and SahH.

ELISAs have been developed for the serological diagnosis of bTB as a supplementary strategy using various recombinant proteins (MPB70, MPB83, and ESAT6) as well as native antigens (P22, $20 \mathrm{kDa}$, CMP, and PPD) [1, 2, 5, 12, 17]. Initially, crude and purified native proteins were evaluated as candidate bTB ELISA antigens, such as MPB70, MPB64, MPB59, P32, P70, P65, and CMP70 [17-21]. Purified native antigens, such as CMP, 20 kDa, and MPB70, exhibit higher sensitivity and specificity than those of crude native antigens (PPD). In this study, the native 20-kDa antigen showed higher sensitivity and specificity than those of PPD. Immunospecific-recombinant proteins of M. bovis have been applied as bTB ELISA antigens, such as MPB70, MPB83, ESAT6, CFP10, Mb0143, PE5, PE13, TB10.4, TB15.3, Rv3615c, Rv3020c, and ESAT6/MPB70/MPB83 (fusion protein) [12, 22-26]. In this study, we newly mixed MPB70 with SahH as a recombinant antigen. It was detected as an immunoreactive protein group by 2D-gel and immunoblot analyses. SahH encodes S-adenosylhomocysteinase, an enzyme that catalyses the reversible hydrolysis 
of S-adenosylhomocysteine to homocysteine and adenosine and is involved in the mycobacterial stress response [27, 28]. SahH enhances attachment to IL-8 and promotes entry into neutrophils [29]. However, the serological characteristics of SahH have not been reported to date. In this study, the antigenicity of recombinant SahH was confirmed with a size of $75 \mathrm{kDa}$ determined by western blotting (Fig. 1c). Reported values for the sensitivity and specificity of recombinant proteins are $63.0-83.2 \%$ and $75.5-98.0 \%$, respectively $[12,22,25,26]$. In this study, rM70S showed higher or equivalent sensitivity and specificity than those in previous studies. The antigen combination is an important determinant of the sensitivity and specificity of ELISA. In this study, rM70S exhibited comparable sensitivity and specificity to those of the native $20 \mathrm{kDa}$ ELISA antigen. Therefore, rM70S is an appropriate antigen combination with easy and simple production and standardisation.

\section{Conclusions}

Even though PPD showed the lowest specificity and sensitivity among the three ELISA antigens evaluated in this study, these values were acceptable for herd screening. PPD has been used as an IST reagent and can easily be applied to bTB ELISA. Overall, native 20K ELISA exhibited higher sensitivity and specificity than those of rM70S. However, rM70S is more convenient for commercialisation with respect to mass production, standardisation, and known concentrations $[17,25]$.

bTB ELISA using purified and recombinant antigens will be useful for individual tests as well as herd screening [1, 2, 26]. In slaughterhouses, randomly selected serum samples can be evaluated, followed by the tracking of antibodypositive reactors $[12,22,30]$. For the control of bTB, ELISA will decrease labour and time requirements. Furthermore, HUMID can account for the gap in the diagnostic window of CEMID. Therefore, the combination of HUMID and CEMID provides a comprehensive diagnostic system for bTB.

\section{Methods}

\section{Purified protein derivative}

Mycobacterium bovis AN5 was cultured on Sauton broth to harvest purified protein derivative (PPD), which was produced following the national standard protocol [11]. Briefly, after culture for 8 weeks at $37^{\circ} \mathrm{C}$, the sample was heated at $100{ }^{\circ} \mathrm{C}$ for $3 \mathrm{~h}$ and the supernatant was harvested by ultracentrifugation to remove bacteria. The culture supernatant was precipitated by $40 \%$ trichloroacetic acid (TCA). The precipitate was washed with $1 \%$ TCA, acetone, and ethyl ether. The washed pellet was dried at $37^{\circ} \mathrm{C}$. The protein concentration was determined using a BCA Protein Assay Kit (Pierce, Waltham, MA, USA). PPD was aliquoted at $1 \mathrm{mg} / \mathrm{vial}$, dried, and stored at $-20^{\circ} \mathrm{C}$.

\section{Purification of the 20-kDa antigen}

The 20-kDa protein was purified from the culture supernatant of M. bovis AN5 after killing by heating using a MonoQ HR 5/5 column with an NaCl gradient with AKTA explorer (GE Healthcare, Chicago, IL, USA) for anion exchange chromatography [5]. The culture supernatant was loaded onto the MonoQ HR 5/5 column and fractionated by $1.0 \mathrm{~mL} / \mathrm{min}$ of a linear gradient from 0 to $2.0 \mathrm{M} \mathrm{NaCl}$ using buffer $\mathrm{A}(30 \mathrm{mM}$ Tris- $\mathrm{HCl}$ with $2 \%$ butanol, $\mathrm{pH} 8.8)$ and buffer $\mathrm{B}$ (buffer $\mathrm{A}$ with $2.0 \mathrm{M} \mathrm{NaCl}$ ) at a flow rate of $1.0 \mathrm{~mL} / \mathrm{min}$. The protein concentration, as determined by the optical density (OD) at $280 \mathrm{~nm}$, salt concentration, and fraction count were recorded. Fractions with absorbance values of $>1.0$ at $280 \mathrm{~nm}$ were further analysed by the BCA protein assay (Pierce), SDS-PAGE, and western blotting. The fractions corresponding to $20 \mathrm{kDa}$ were collected, mixed, and stored at $-20^{\circ} \mathrm{C}$.

\section{Recombinant MPB70 and SahH}


The plasmid pGS (Bionote Co., Hwaseong, Republic of Korea), Escherichia coli Top10F', and E. coli BL21 competent cells were prepared and stocked. The primers were designed according to the sequences of genes encoding MPB70 and SahH of the M. bovis AF2122/97 strain (GenBank accession number NC002945) and synthesised. Recombinant MPB70 and SahH proteins were expressed in E. coli BL21. Genomic DNA from M. bovis AN5 was isolated by the GuSCN/silica method. The MPB70 and SahH DNA fragments were amplified from AN5 genomic DNA by polymerase chain reaction (PCR). The amplified DNA fragments were purified using the Geneclean Kit (Bio 101, Inc., La Jolla, CA, USA), digested with the appropriate restriction enzyme, cloned into the pGS vector, and transformed into the E. coli strain Top10F'. The positive clones were screened by colony PCR and sequenced using vector primers. Each MPB70 and SahH plasmid was transformed into E. coli BL21. Transformed E. coli cells were inoculated in LB and cultivated overnight at $37^{\circ} \mathrm{C}$ in a shaker. When the $A 600$ value reached 1.0, isopropyl- $\beta$-d-thiogalactoside was added to a final concentration of $2.5 \mathrm{mM}$. The mixture was grown for another $5 \mathrm{~h}$. The cells were harvested by centrifugation at $5,000 \times$ $\mathrm{g}$ for $15 \mathrm{~min}$ at $4^{\circ} \mathrm{C}$. The protein was purified on a DEAE-Sepharose gel after harvesting inclusion bodies by ultrasonication. Each fraction was examined by SDS-PAGE and western blotting.

\section{SDS-PAGE and western blotting}

PPD, MPB70, SahH, and 20-kDa proteins were analysed by SDS-PAGE and western blotting [5]. Equal volumes of serum samples $(n=3)$ from a bTB-free farm (confirmed by annual ISTs and the lack of clinical signs of infection) were mixed and used as a negative control. Mycobacterium bovis AN5-positive serum $(n=1)$ was used as a positive control. Each serum sample was diluted as 1:500 for western blotting. The protein (10 $\mu \mathrm{g}$ per lane) was separated by SDS-PAGE and transferred onto a nitrocellulose (NC) membrane soaked in $600 \mathrm{~mL}$ of transfer buffer (15.6 mM Tris and $120 \mathrm{mM}$ glycine) plus $150 \mathrm{~mL}$ methanol at $100 \mathrm{~V}$ and $400 \mathrm{~mA}$ for $1 \mathrm{~h} \mathrm{[5].} \mathrm{The} \mathrm{membranes} \mathrm{were} \mathrm{then} \mathrm{incubated} \mathrm{in} \mathrm{M.} \mathrm{bovis-}$ infected cattle serum diluted $1: 500$ in $0.01 \mathrm{M} \mathrm{PBS}$ at $37^{\circ} \mathrm{C}$ for $1 \mathrm{~h}$, followed by anti-bovine IgG horseradish peroxidase conjugate (Kirkegaard \& Perry Laboratories Inc., Gaithersburg, MD, USA) diluted 1:1,000 in $0.01 \mathrm{M}$ PBS at $37^{\circ} \mathrm{C}$ for $1 \mathrm{~h}$. After washing with 0.01 M PBS (pH 7.4), the membranes were incubated in 3,3'-diaminobenzidine (Sigma, St. Louis, $\mathrm{MO}$, USA) for $5 \mathrm{~min}$ at room temperature $\left(22 \pm 2{ }^{\circ} \mathrm{C}\right)$.

\section{ELISA}

Eighteen serum samples from M. bovis-positive cattle at 8 farms (confirmed by ISTs and the isolation of M. bovis) and 975 serum samples from M. bovis-free cattle at 14 farms (confirmed by ISTs and the lack of clinical signs of infection) were subjected to testing by PPD, MPB70/SahH, and 20-kDa ELISA. Diagnostic efficiency was compared based on sensitivities and specificities. Positive control and negative control sera were used for each ELISA for validation. ELISA results were analysed according to three criteria, the OD value, S/N ratio, and S/P ratio $[1,17,22,23]$. The optimal method for effectively diagnosing bovine tuberculosis was determined by comparisons of the sensitivity and specificity of each parameter.

\section{Statistical analysis}

ELISA results were analysed by Student's t-tests. All data are presented as means \pm standard deviations (error bars). All statistical values were considered significant at $\mathrm{P} \leq 0.05$.

\section{Abbreviations}

bTB: Bovine tuberculosis; PPD: Purified protein derivative; ELISA: Enzyme-linked immunosorbent assay; IST: Intradermal skin test; CEMID: Cell-mediated immunity-based diagnosis; HUMID: Humoral immunity-based diagnosis; CFP: Culture filtrate proteins; INS: Insoluble proteins; SOL: Soluble proteins; S/P: Sample/positive; S/N: 
Sample/negative; PPV: Positive predictive value; NPV: Negative predictive value; ROC: Receiver operating characteristic; PCR: polymerase chain reaction

\section{Declarations}

\section{Ethics approval and consent to participate}

This study was approved by the Animal and Plant Quarantine Agency's Institutional Animal Care and Use Committee, Republic of Korea. Verbal informed consent for the study was obtained from all owners prior to sample collection from the cattle.

\section{Consent for publication}

Not applicable

\section{Availability of data and materials}

The data that support the findings of this study are available from the corresponding author upon reasonable request.

\section{Competing interests}

The authors declare that they have no competing interests.

\section{Funding}

This study was funded by the Animal and Plant Quarantine Agency (APQA). The funders had no role in the design of the study, data collection, analyses, interpretation of data, or preparation of the manuscript.

\section{Authors' contributions}

YSC conceived of the study. YSC, SEL, J-TW, JO, HWC, JHK, J-TK, GH, and CP performed the experiments. SJ and JMK helped with data analysis. YSC, SJ and JMK interpreted the results and YSC wrote the article. All authors read and approved the final manuscript.

\section{Acknowledgments}

The authors would like to thank Editage (www.editage.co.kr) for English language editing.

\section{Authors details}

${ }^{1}$ Bacterial Disease Division, Department of Animal and Plant Health Research, Animal and Plant Quarantine Agency, Gimcheon, Gyeongsangbuk-do, Republic of Korea

${ }^{2}$ Gyeonggi Province Veterinary Service Center, Suwon, Gyeonggi-do, Republic of Korea

${ }^{3}$ Bionote Co., LTD., 22 Samseong 1-ro 4-gil, Hwaseong-si, Gyeonggi-do, Republic of Korea

${ }^{4}$ ChoongAng Vaccine Laboratory (CVL), Daejeon, Republic of Korea

\section{References}


1. Infantes-Lorenzo JA, Moreno I, Roy A, Risalde MA, Balseiro A, de Juan L, Romero B, Bezos J, Puentes E, Akerstedt J et al: Specificity of serological test for detection of tuberculosis in cattle, goats, sheep and pigs under different epidemiological situations. BMC Vet Res 2019, 15(1):70.

2. Garbaccio SG, Garro CJ, Delgado F, Tejada GA, Eirin ME, Huertas PS, Leon EA, Zumarraga MJ: Enzyme-linked immunosorbent assay as complement of intradermal skin test for the detection of mycobacterium bovis infection in cattle. Tuberculosis (Edinb) 2019, 117:56-61.

3. Jeon HS, Shin AR, Son YJ, Kim JM, Jang Y, Kim S, Lee KI, Choi CH, Park JK, Kim HJ: An evaluation of the use of immunoglobulin A antibody response against mycobacterial antigens for the diagnosis of Mycobacterium bovis infection in cattle. J Vet Diagn Invest 2015, 27(3):344-351.

4. Jeon BY, Kim SC, Je S, Kwak J, Cho JE, Woo JT, Seo S, Shim HS, Park BO, Lee SS et al: Evaluation of enzymelinked immunosorbent assay using milk samples as a potential screening test of bovine tuberculosis of dairy cows in Korea. Res Vet Sci 2010, 88(3):390-393.

5. Cho YS, Lee SE, Ko YJ, Cho D, Lee HS, Hwang I, Nam H, Heo E, Kim JM, Jung S: Definition of purified enzymelinked immunosorbent assay antigens from the culture filtrate protein of Mycobacterium bovis by proteomic analysis. J Immunoassay Immunochem 2009, 30(3):291-304.

6. Luciano SA, Roess A: Human zoonotic tuberculosis and livestock exposure in low- and middle-income countries: A systematic review identifying challenges in laboratory diagnosis. Zoonoses Public Health 2020, 67(2):97-111.

7. Cadmus S, Akinseye VO, van Soolingen D: Mycobacterium bovis in humans and M. tuberculosis in animals in Nigeria: an overview from 1975-2014. Int J Tuberc Lung Dis 2019, 23(11):1162-1170.

8. Siala M, Cassan C, Smaoui S, Kammoun S, Marouane C, Godreuil S, Hachicha S, Mhiri E, Slim L, Gamara D et al: A first insight into genetic diversity of Mycobacterium bovis isolated from extrapulmonary tuberculosis patients in South Tunisia assessed by spoligotyping and MIRU VNTR. PLoS Negl Trop Dis 2019, 13(9):e0007707.

9. Robinson PA: Farmer and veterinarian attitudes towards the risk of zoonotic Mycobacterium bovis infection in Northern Ireland. Vet Rec 2019, 185(11):344.

10. Diallo M, Diarra B, Sanogo M, Togo AC, Somboro AM, Diallo MH, Traore B, Maiga M, Kone Y, Tounkara K et al: Molecular identification of Mycobacterium bovis from cattle and human host in Mali: expanded genetic diversity. BMC Vet Res 2016, 12(1):145.

11. Cho YS, Jang YB, Lee SE, Cho JY, Ahn JM, Hwang I, Heo E, Nam HM, Cho D, Her M et al: Short communication: Proteomic characterization of tuberculin purified protein derivative from Mycobacterium bovis. Res Vet Sci 2015, 101:117-119.

12. Souza IIF, Rodrigues RA, Goncalves Jorge KS, Silva MR, Lilenbaum W, Vidal CES, Etges RN, Kostovic M, Araujo FR: ELISA using a recombinant chimera of ESAT-6/MPB70/MPB83 for Mycobacterium bovis diagnosis in naturally infected cattle. J Vet Med Sci 2019, 81(1):9-14.

13. Bezos J, Casal C, Romero B, Schroeder B, Hardegger R, Raeber AJ, Lopez L, Rueda P, Dominguez L: Current antemortem techniques for diagnosis of bovine tuberculosis. Res Vet Sci 2014, 97 Suppl:S44-52.

14. Whelan C, Shuralev E, Kwok HF, Kenny K, Duignan A, Good M, Davis WC, Clarke J: Use of a multiplex enzymelinked immunosorbent assay to detect a subpopulation of Mycobacterium bovis-infected animals deemed negative or inconclusive by the single intradermal comparative tuberculin skin test. J Vet Diagn Invest 2011, 23(3):499-503.

15. de la Rua-Domenech R, Goodchild AT, Vordermeier HM, Hewinson RG, Christiansen KH, Clifton-Hadley RS: Ante mortem diagnosis of tuberculosis in cattle: a review of the tuberculin tests, gamma-interferon assay and other ancillary diagnostic techniques. Res Vet Sci 2006, 81(2):190-210. 
16. Amadori M, Lyashchenko KP, Gennaro ML, Pollock JM, Zerbini I: Use of recombinant proteins in antibody tests for bovine tuberculosis. Vet Microbiol 2002, 85(4):379-389.

17. Cho YS, Jung SC, Kim JM, Yoo HS: Enzyme-linked immunosorbent assay of bovine tuberculosis by crude mycobacterial protein 70. J Immunoassay Immunochem 2007, 28(4):409-418.

18. Fifis T, Plackett P, Corner LA, Wood PR: Purification of a major Mycobacterium bovis antigen for the diagnosis of bovine tuberculosis. Scand J Immuno/ 1989, 29(1):91-101.

19. Harboe M, Wiker HG, Duncan JR, Garcia MM, Dukes TW, Brooks BW, Turcotte C, Nagai S: Protein G-based enzymelinked immunosorbent assay for anti-MPB70 antibodies in bovine tuberculosis. J Clin Microbio/ 1990, 28(5):913921.

20. Fifis T, Costopoulos C, Corner LA, Wood PR: Serological reactivity to Mycobacterium bovis protein antigens in cattle. Vet Microbiol 1992, 30(4):343-354.

21. Wood PR, Corner LA, Rothel JS, Ripper JL, Fifis T, McCormick BS, Francis B, Melville L, Small K, de Witte K et al: A field evaluation of serological and cellular diagnostic tests for bovine tuberculosis. Vet Microbiol 1992, 31(1):7179.

22. Fontana S, Pacciarini M, Boifava M, Pellesi R, Casto B, Gastaldelli M, Koehler H, Pozzato N, Casalinuovo F, Boniotti MB: Development and evaluation of two multi-antigen serological assays for the diagnosis of bovine tuberculosis in cattle. J Microbiol Methods 2018, 153:118-126.

23. Trost B, Stuber T, Surujballi O, Nelson J, Robbe-Austerman S, Smith NH, Desautels L, Tikoo SK, Griebel P: Investigation of the cause of geographic disparities in IDEXX ELISA sensitivity in serum samples from Mycobacterium bovis-infected cattle. Sci Rep 2016, 6:22763.

24. Waters WR, Palmer MV, Stafne MR, Bass KE, Maggioli MF, Thacker TC, Linscott R, Lawrence JC, Nelson JT, Esfandiari J et al: Effects of Serial Skin Testing with Purified Protein Derivative on the Level and Quality of Antibodies to Complex and Defined Antigens in Mycobacterium bovis-Infected Cattle. Clin Vaccine Immunol 2015, 22(6):641-649.

25. Waters WR, Buddle BM, Vordermeier HM, Gormley E, Palmer MV, Thacker TC, Bannantine JP, Stabel JR, Linscott R, Martel $\mathrm{E}$ et al: Development and evaluation of an enzyme-linked immunosorbent assay for use in the detection of bovine tuberculosis in cattle. Clin Vaccine Immunol 2011, 18(11):1882-1888.

26. Souza, II, Melo ES, Ramos CA, Farias TA, Osorio AL, Jorge KS, Vidal CE, Silva AS, Silva MR, Pellegrin AO et al: Screening of recombinant proteins as antigens in indirect ELISA for diagnosis of bovine tuberculosis. Springerplus 2012, 1(1):77.

27. Viswanathan G, Joshi SV, Sridhar A, Dutta S, Raghunand TR: Identifying novel mycobacterial stress associated genes using a random mutagenesis screen in Mycobacterium smegmatis. Gene 2015, 574(1):20-27.

28. Singhal A, Arora G, Sajid A, Maji A, Bhat A, Virmani R, Upadhyay S, Nandicoori VK, Sengupta S, Singh Y: Regulation of homocysteine metabolism by Mycobacterium tuberculosis S-adenosylhomocysteine hydrolase. Sci Rep 2013, 3:2264.

29. Dziadek B, Brzostek A, Grzybowski M, Fol M, Krupa A, Kryczka J, Plocinski P, Kurdowska A, Dziadek J: Mycobacterium tuberculosis AtsG (Rv0296c), GImU (Rv1018c) and SahH (Rv3248c) Proteins Function as the Human IL-8-Binding Effectors and Contribute to Pathogen Entry into Human Neutrophils. PLoS One 2016, 11(2):e0148030.

30. Welby S, Govaerts M, Vanholme L, Hooyberghs J, Mennens K, Maes L, Van Der Stede Y: Bovine tuberculosis surveillance alternatives in Belgium. Prev Vet Med 2012, 106(2):152-161.

\section{Figures}

Page $10 / 13$ 
a
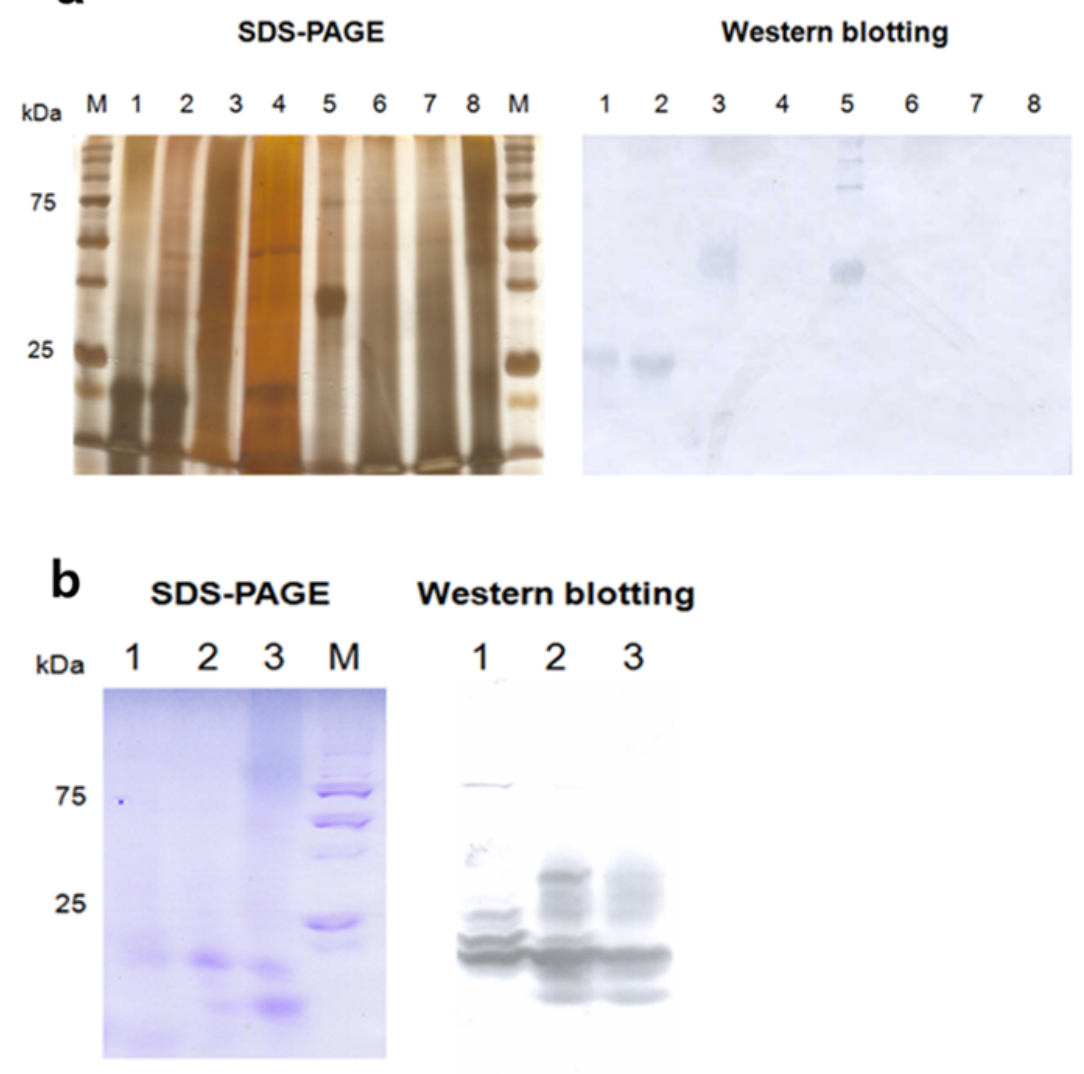

C

SDS-PAGE

Western blotting

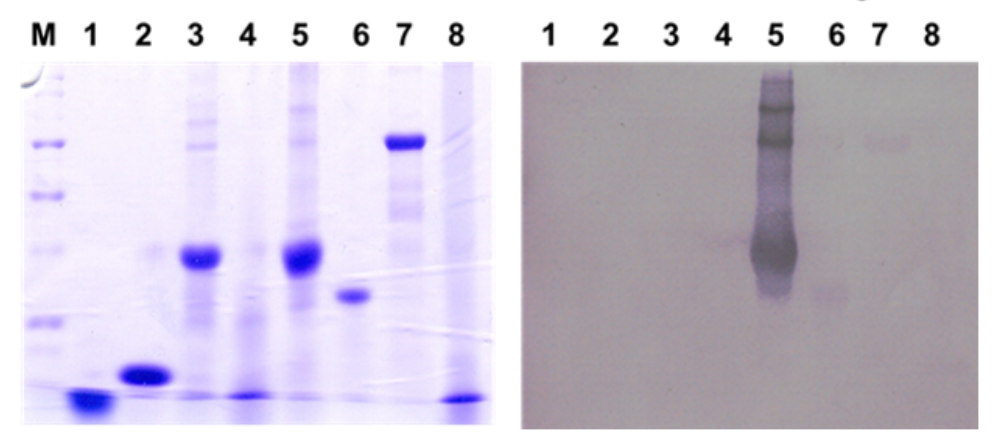

Figure 1

SDS-PAGE and Western blotting of PPD, 20kDa, MPB70, and SahH proteins. In (a), PPDs, CFP, INS, SOL, and MPB70 were produced from $M$. bovis. Johnin and PPDs were produced from M. avium, and PPD from M. phlei. M. protein marker, lane 1. M. bovis PPD, lane 2. M. bovis CFP, lane 3. M. bovis INS, lane 4. M. bovis SOL, lane 5. Recombinant MPB70, lane 6. M. avium Johnin, lane 7. M. avium PPD, lane 8. M. phlei PPD. In (b), native 20 kDa was analyzed by SDS-PAGE and Western blotting. Lane 1. APQA 20 kDa, lane 2. CVL 20 kDa, lane 3. M. bovis PPD, M. protein marker. In (c), With recombinant MPB70, ESAT6, HspX, PhoS, MPB64, Antigen 85, SahH, and native PPD from M. bovis were analyzed. M. protein marker, lane 1. ESAT6, lane 2. HspX, lane 3. PhoS, lane 4. MPB64, lane 5. MPB70, lane 6. Antigen 85, lane 7. SahH, lane 8. M. bovis PPD. 
a

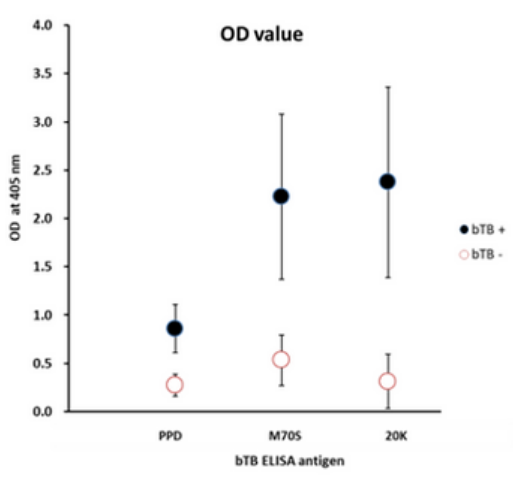

\section{b}

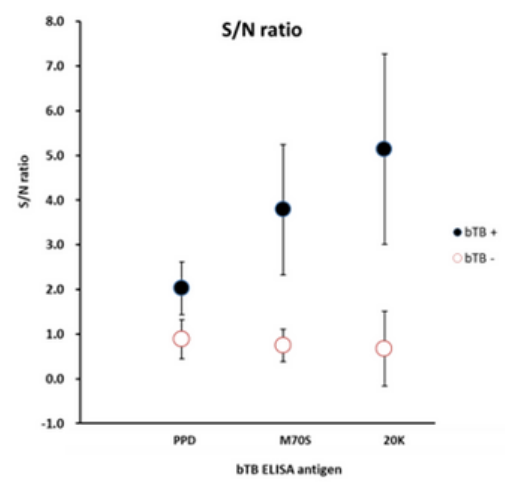

c

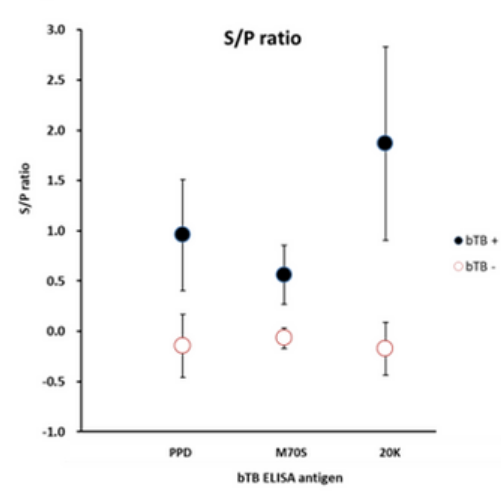

Figure 2

Comparison of OD value (a), S/N ratio (b), and S/P ratio (c) of PPD, M70S, and 20K ELISA. bTB+ was M. bovis-infected cattle's sera $(n=18)$, and bTB- M.bovis-negative cattle's sera in PPD ELISA $(n=975)$, M70S ELISA $(n=174)$, and 20K ELISA ( $n=954)$. 
a

OD value

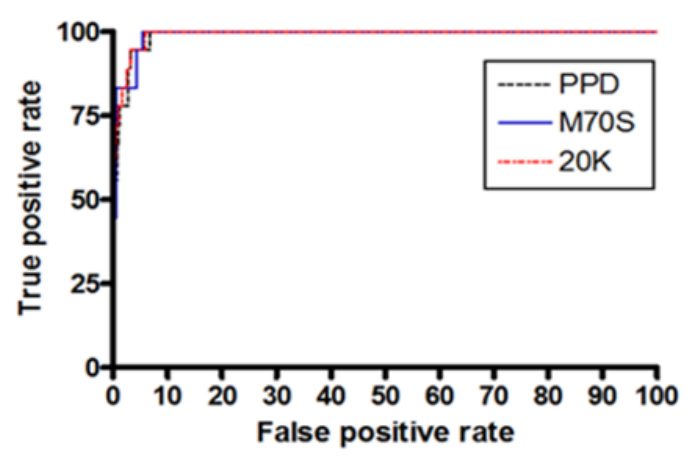

b

$\mathrm{S} / \mathrm{N}$ ratio

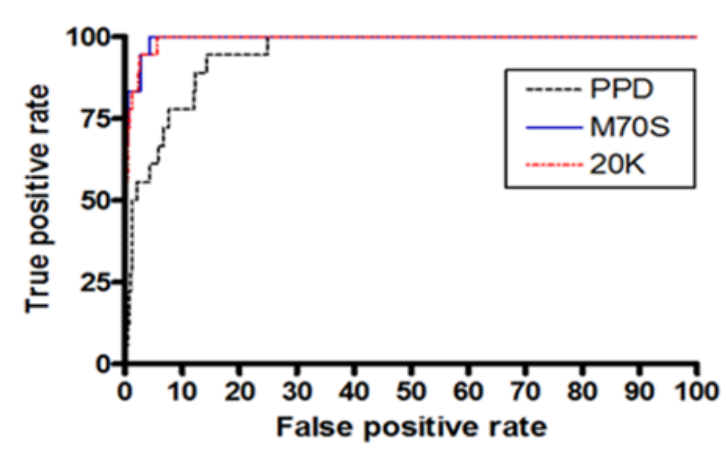

C

S/P ratio

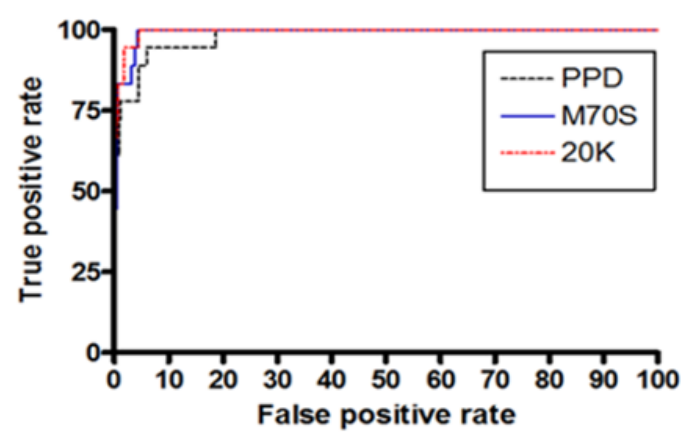

Figure 3

Comparison of ROC curve of PPD, M70S, and 20K ELISA. Criteria of cutoff were OD value (a), S/N ratio (b), and S/P ratio (c). 